\title{
Mild Cognitive Impairment and Dementia Show Contrasting Associations with Risk of Cancer
}

\author{
Kimberly D. van der Willik ${ }^{a, b}$ Rikje Ruiter ${ }^{a}$ Frank J. Wolters ${ }^{a, c}$ \\ M. Kamran Ikram ${ }^{a}$ c Bruno H. Stricker ${ }^{a}$ Michael Hauptmann ${ }^{b}$ \\ Annette Compter $^{d}$ Sanne B. Schagen ${ }^{b}$ M. Arfan Ikram ${ }^{a}$
}

${ }^{a}$ Department of Epidemiology, Erasmus Medical Center, Rotterdam, The Netherlands; ${ }^{\mathrm{b}}$ Department of Psychosocial

Research and Epidemiology, Netherlands Cancer Institute, Amsterdam, The Netherlands; ' ${ }^{\text {Department }}$ of

Neurology, Erasmus Medical Center, Rotterdam, The Netherlands; ${ }^{\mathrm{d}}$ Department of Neuro-Oncology, Netherlands

Cancer Institute, Amsterdam, The Netherlands

\section{Keywords}

Dementia $\cdot$ Alzheimer's disease $\cdot$ Mild cognitive

impairment · Cancer · Epidemiology

\begin{abstract}
Background: To investigate and to compare the relation between dementia and cancer with the association between mild cognitive impairment ( $\mathrm{MCl}$ ) and cancer. Methods: A total of 13,207 persons from the Rotterdam Study were followed between 1990 and 2013 for the onset of dementia and cancer (sample 1). Between 2002 and 2005, a subset of 5,181 persons underwent extensive cognitive testing for $\mathrm{MCl}$ and subsequently were followed up for cancer until 2013 (sample 2). We used Cox proportional hazard models to determine the association between dementia and cancer, and $\mathrm{MCl}$ and cancer. Results: In sample 1, 1,404 patients were diagnosed with dementia, and 2,316 developed cancer (63 among dementia cases). Dementia was associated with a decreased risk of cancer (hazard ratio [HR] $0.53 ; 95 \% \mathrm{Cl} 0.41-$ 0.68). In sample 2,513 persons were diagnosed with $\mathrm{MCl}$ and
\end{abstract}

670 persons developed cancer ( 81 among $\mathrm{MCl}$ cases). In contrast to individuals with dementia, those with $\mathrm{MCl}$ tended to have an increased risk of cancer (HR 1.25; 95\% Cl 0.99-1.58). Conclusions: We found that persons with $\mathrm{MCl}$ tended to have an increased risk of cancer, whereas those with dementia have a decreased risk. These findings call into question a biological explanation for the inverse link between dementia and cancer, thereby suggesting the presence of methodological bias.

(C) 2018 The Author(s)

Published by S. Karger AG, Basel

\section{Introduction}

Dementia, including Alzheimer disease (AD), and cancer are global health priorities. Interestingly, several studies have consistently shown an inverse link between the 2 diseases. Patients with dementia have a decreased risk of cancer [1-7], while persons with a history of cancer are affected less often from subsequent dementia $[1,2,4$, $5,8]$. 
Different biological mechanisms underlying this inverse association have been proposed, including pathways of cell proliferation and cell survival $[9,10]$. Furthermore, epigenetic processes including DNA methylation have been considered contributing to this inverse association. Yet, patients with dementia are less likely to be screened for other diseases and have a limited life expectancy, both potentially contributing to a decreased subsequent incidence of cancer. Therefore, methodological bias, such as surveillance bias and survival bias, possibly explaining the inverse link between dementia and cancer, has so far not been satisfactorily ruled out.

If indeed a biological mechanism underlies the association between dementia and cancer, this would likely extend across the different preclinical stages of cognitive impairment. Mild cognitive impairment (MCI) is often considered the transitional stage between normal cognition and dementia, although not all cases of MCI ultimately lead to dementia $[11,12]$. As such, it is considered an early clinical manifestation of the same pathological processes that underlie dementia and AD. Accordingly, we hypothesized that if the inverse link between dementia and cancer is truly biologically determined, this should be also reflected in the association between MCI and cancer.

We therefore investigated and compared the association between dementia and cancer with the association between MCI and cancer.

\section{Materials and Methods}

\section{Setting}

This study is embedded in the Rotterdam Study, a populationbased prospective cohort that started in 1990 in the Netherlands. The initial cohort (RS-I) consisted of 7,983 participants (78\% of invitees) aged 55 years or older residing in the district Ommoord in Rotterdam. The second cohort (RS-II) started in 2000 and was composed of 3,011 participants (67\% of invitees) in the same district who had turned 55 years or moved into this area. The third cohort (RS-III) was started in 2006, in which 3,392 participants (65\% of invitees) were included. The design of the Rotterdam Study has been described in detail previously [13].

Standard Protocol Approvals, Registrations, and Patient Consents

The Rotterdam Study was approved by the Medical Ethics Committee of Erasmus Medical Center and by the board of The Netherlands Ministry of Health, Welfare, and Sports. A written informed consent was obtained from all participants.

\section{Study Population}

For the current study, 2 partly overlapping samples from the Rotterdam Study were defined. First, in sample 1, we investigated the association between dementia and risk of cancer, using demen- tia as a time-varying exposure. This analysis used the complete sample and follow-up of the Rotterdam Study. Of 14,926 study participants, we excluded patients with prevalent dementia $(n=$ 527), participants who were not sufficiently screened for dementia $(n=743)$, and participants with prevalent cancer $(n=449)$, leaving a total of 13,207 persons (Fig. 1a).

Second, in sample 2, we investigated the association between MCI and risk of cancer, using MCI at a single assessment, since assessment of incident MCI is more difficult than incident dementia in a population-based setting due to limited information about the date of onset. This sample originated from the fourth follow-up round of RS-I, the second round of RS-II, and the first round of the RS-III. In total, 9,065 participants were assessed for MCI, of whom we excluded patients with prevalent dementia $(n=$ $124)$, persons not sufficiently screened for dementia $(n=283)$, not sufficiently screened for MCI $(n=326)$, or aged below 60 years $(n=$ $2,599)$. In addition, participants with prevalent cancer $(n=214)$ or incident cancer before MCI assessment $(n=338)$ were excluded, resulting in 5,181 participants for the MCI analysis (Fig. 1). To enhance comparability between the analyses for dementia and MCI, we additionally performed a comparative analysis between dementia and cancer in sample 2 by using a single assessment of prevalent dementia $(n=124)$. Persons with MCI $(n=513)$ were excluded for this analysis (Fig. 1b).

\section{Ascertainment of Incident Dementia}

Participants were screened for dementia at baseline and subsequent center visits with the Mini-Mental State Examination and the Geriatric Mental Schedule organic level [14]. Those with a Mini-Mental State Examination score $<26$ or Geriatric Mental Schedule score $>0$ underwent further investigation and informant interview, including the Cambridge Examination for Mental Disorders of the Elderly. During each center visit, all participants also underwent routine cognitive assessment, including a verbal fluency test [Word Fluency Test (WFT), animal categories], 15-word learning test, letter-digit substitution task (LDST), Stroop test, and Purdue pegboard task. In addition, the entire cohort was continuously under surveillance for dementia through electronic linkage of the study database with medical records from general practitioners and the regional institute for outpatient mental health care. Available information on clinical neuroimaging was used when required for diagnosis of dementia subtype. A consensus panel led by a consultant neurologist established the final diagnosis according to standard criteria for dementia (DSM-III-R, Diagnostic and Statistical Manual of Mental Disorders, Third Edition, Revised), AD (NINCDS-ADRDA, National Institute of Neurological and Communicative Disorders and Stroke and the Alzheimer's Disease and Related Disorders Association), and vascular dementia (NINDS-AIREN, National Institute of Neurological Disorders and Stroke and the Association Internationale pour la Recherché et l'Enseignement en Neurosciences). Follow-up until January 1, 2013 was virtually complete (92.4\% of potential person-years).

\section{Assessment of MCI}

Extensive cognitive testing for MCI assessment was implemented in the Rotterdam Study between 2002 and 2005, which encompasses the fourth examination round of RS-I, the second examination round of RS-II and the first examination round of RS-III. MCI was defined as the presence of self-perceived cogni- 
Sample 1

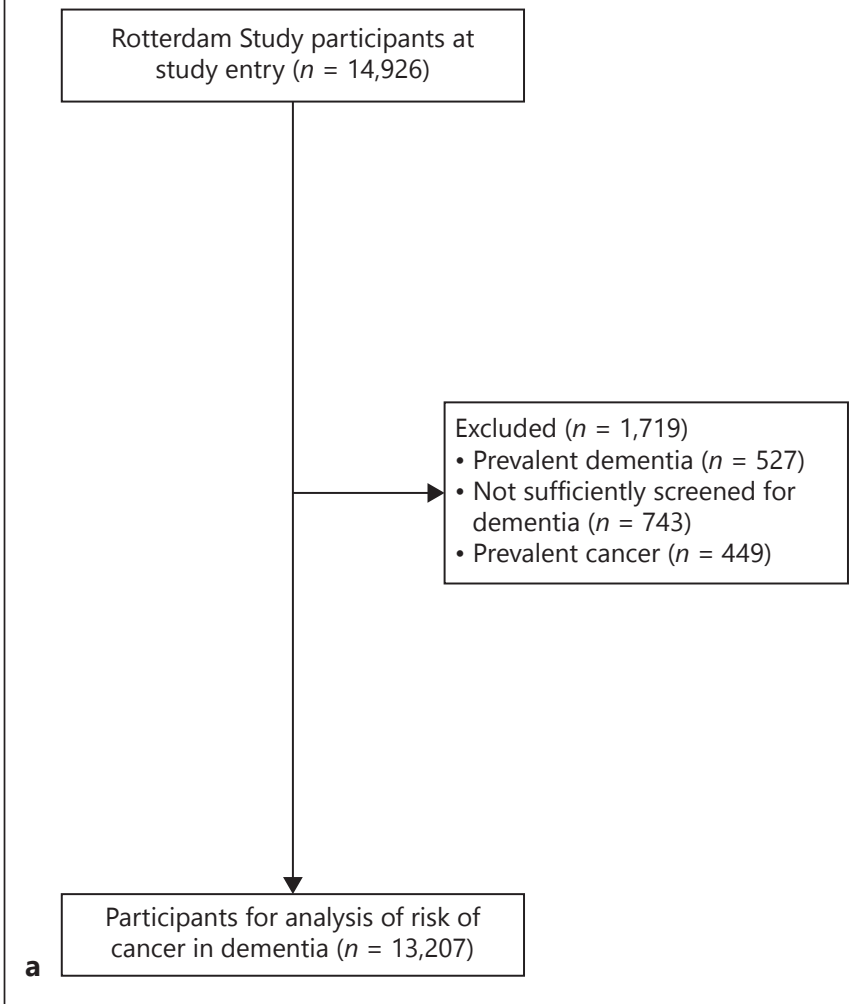

Sample 2

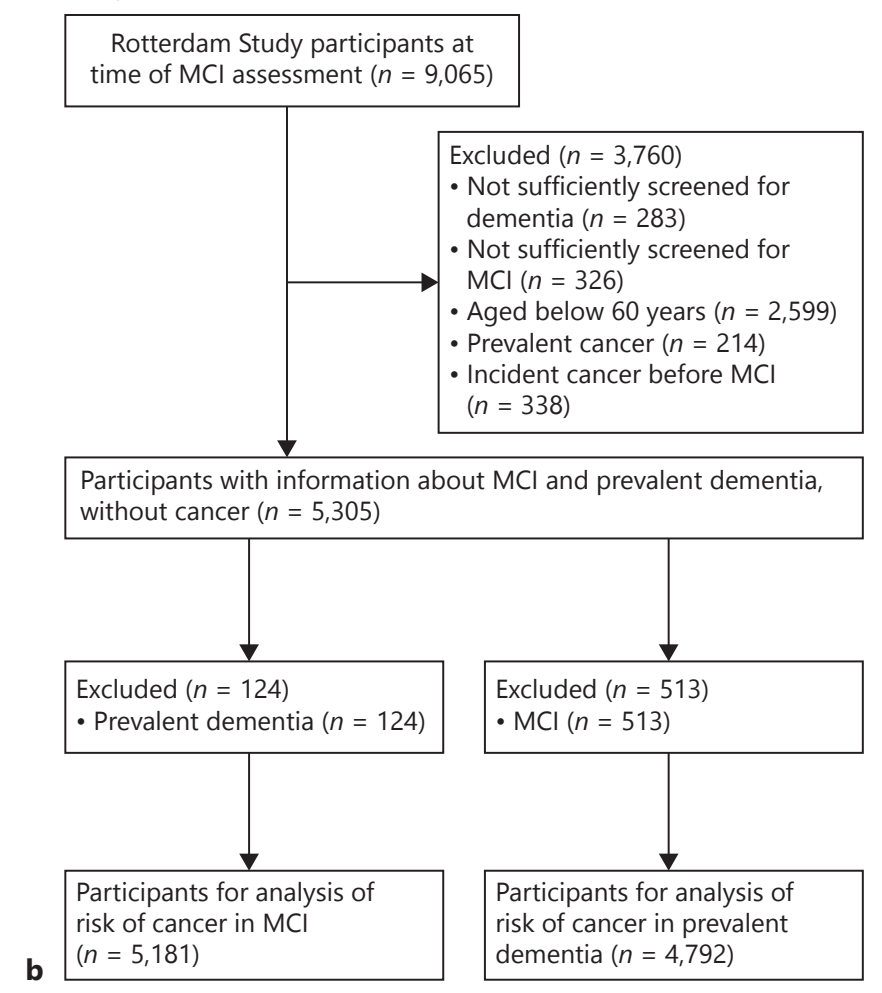

Fig. 1. a, b Flowchart of participants in samples 1 and 2. The association between incident dementia and cancer was studied in sample 1. For sample 1, all participants of the Rotterdam Study were included at study entry, that is, the first rounds of the first (RS-I), second (RS-II), and third cohort (RS-III). In total, sample 1 consisted of 13,207 participants. Sample 2 originated from the fourth follow-up round of RS-I, the second round of RS-II, and the

tive complaints (defined as at least one of 6 questions on memory and daily functioning) and cognitive impairment as assessed with neuropsychological tests in the absence of dementia, in persons aged $\geq 60$ years [15]. The neuropsychological tests measured memory function (15-word learning test immediate and delayed), information-processing speed (LDST, Stroop first and second subtask), and executive functioning (LDST, Stroop third subtask, and WFT).

MCI was classified as amnestic (impaired scores on memory function irrespective of other domains) and non-amnestic MCI (normal memory function but impaired score on informationprocessing speed or executive function).

\section{Assessment of Incident Cancer}

The primary outcome of interest was the incidence of cancer. Two research physicians independently assessed the diagnosis of cancer based on medical records obtained through general practitioners and hospital discharge letters. Additional information was collected through linkage with the Dutch Hospital Data, National Can- first round of RS-III. In this sample, the association between MCI and cancer was investigated after excluding participants with prevalent dementia, since the absence of dementia is part of the definition of MCI. In addition, a comparative analysis was performed in sample 2 investigating the risk of cancer in patients with prevalent dementia. For this comparative analysis, persons with MCI were excluded. MCI, mild cognitive impairment.

cer Registry, and Dutch pathology database (PALGA). Only cases confirmed by pathology were used. Cancer was classified according to the International Classification of Diseases tenth edition. In case of discrepancy, consensus was sought through consultation with a cancer epidemiologist. Follow-up of cancer registration was completed up to January 1, 2013. Non-melanoma skin cancers (NMSC) were not included in the definition of cancer for the analysis.

\section{Other Assessments}

Baseline was study entry for sample 1 and time of MCI assessment for sample 2. Education level (primary: primary education, lower: lower general education, intermediate general education, or lower vocational education, intermediate: intermediate vocational education or higher general education, or higher: higher vocational education or university), smoking status (current, former, never), alcohol use (yes, no), and psycholeptic drug use (yes, no) were assessed at baseline by interview. Body mass index (BMI; $\mathrm{kg} / \mathrm{m}^{2}$ ) was computed from measurements of height and weight. 
Table 1. Characteristics of study population for dementia and MCI

\begin{tabular}{|c|c|c|c|c|}
\hline \multirow[t]{2}{*}{ Characteristic } & \multicolumn{2}{|l|}{ Sample 1} & \multicolumn{2}{|l|}{ Sample 2} \\
\hline & $\begin{array}{l}\text { no dementia } \\
(n=11,803)\end{array}$ & $\begin{array}{l}\text { dementia } \\
(n=1,404)\end{array}$ & $\begin{array}{l}\text { no MCI } \\
(n=4,668)\end{array}$ & $\begin{array}{l}\text { MCI } \\
(n=513)\end{array}$ \\
\hline Age, years & $61.8(12.6)$ & $73.2(11.9)$ & 69.5 (11.7) & $72.0(12.8)$ \\
\hline Gender, females, $n(\%)$ & $6,676(56.6)$ & $996(70.9)$ & $2,732(58.5)$ & $265(51.7)$ \\
\hline $\mathrm{BMI}, \mathrm{kg} / \mathrm{m}^{2}$ & $26.9(4.0)$ & $26.5(3.6)$ & $27.7(4.1)$ & $27.8(4.0)$ \\
\hline \multicolumn{5}{|l|}{ Education level, $n(\%)$} \\
\hline Primary & $1,869(15.8)$ & $430(30.6)$ & $488(10.5)$ & $97(18.9)$ \\
\hline Low & $4,743(40.2)$ & $603(42.9)$ & $2,087(44.7)$ & $214(41.7)$ \\
\hline Intermediate & $3,318(28.1)$ & $289(20.6)$ & $1,392(29.8)$ & $143(27.9)$ \\
\hline High & $1,873(15.9)$ & $82(5.8)$ & $701(15.0)$ & $59(11.5)$ \\
\hline \multicolumn{5}{|l|}{ Smoking status, $n(\%)$} \\
\hline Current & $2,719(23.0)$ & $233(16.6)$ & $609(13.0)$ & $77(15.0)$ \\
\hline Former & $5,395(45.7)$ & $556(39.6)$ & $2,644(56.6)$ & $293(57.1)$ \\
\hline No alcohol use & $1,823(15.4)$ & $304(21.7)$ & $628(13.5)$ & $98(19.1)$ \\
\hline No psycholeptic drug use & $10,410(88.2)$ & $1,142(81.3)$ & $4,065(87.1)$ & $416(81.1)$ \\
\hline
\end{tabular}

Data are presented as $n(\%)$ after imputation.

Age value is median (IQR), BMI value is mean (SD).

MCI, mild cognitive impairment; $n$, number of persons; IQR, interquartile range; BMI, body mass index.

\section{Statistical Analysis}

Cox proportional hazard models were used to study the association between incident dementia and cancer in sample 1. Dementia was used as time-varying variable. In sample 2, we used Cox proportional hazard models investigating the relation between MCI and cancer. All analyses were adjusted for age (continuous), sex, BMI (continuous), education level, smoking status, alcohol use, and psycholeptic drug use. Ethnicity was not used as a confounder since nearly all participants (98\%) were of European descent. Follow-up time started from inclusion in the Rotterdam Study until the date of incident cancer, death, loss to follow-up, or January 1, 2013, whichever came first. Censoring unexposed participants at date of death allowed us to compute cause-specific hazard ratios (HRs), taking into account competing risk of death. To minimize the potential impact of preexisting subclinical malignancy on cognition (i.e., reverse causation), we repeated analyses after excluding the first 2 and 5 years of followup. This was performed in sample 1 by excluding the first 2 and 5 years following study entry for persons free of dementia, and the first 2 and 5 years after dementia diagnosis for dementia patients $[16,17]$. In sample 2 , the first 2 and 5 years after baseline were excluded for both persons with and without MCI. Additionally, we explored effect modification by stratifying for age, sex, and smoking status. In sensitivity analyses, we repeated the analyses using age instead of follow-up time as time scale. The proportional hazards assumption was checked by visual inspection of the Schoenfeld residuals.

To enhance comparability between the dementia and MCI analyses, we performed a Cox proportional hazard analysis in sample 2 to study the risk of cancer in patients with prevalent dementia. In this sample, we additionally censored follow-up time at date of NMSC, stroke, or dementia diagnosis, limiting the effect of possible over- or underdiagnoses of cancer after these conditions.
Finally, direct comparison of the risk of cancer between dementia and MCI was performed by testing whether the HRs of cancer for dementia in sample 1 and 2 differed from the HR of cancer for MCI in sample 2 using a $t$ test.

Missing covariates were imputed using the mean of 5 imputations based on the investigated covariates and outcome. All analyses were performed using IBM SPSS Statistics version 21.0 and the survival package in RStudio version 1.0.44.

\section{Results}

Table 1 shows the characteristics of the study population. Persons who developed dementia during follow-up were at baseline older, were more often women, had a lower BMI, a primary or lower education level, and less often an intermediate or higher education level compared to those not diagnosed with dementia during follow-up. Additionally, persons who developed dementia were less frequently smokers and alcohol users, and used less often psycholeptic drugs. Participants with MCI were older, were more often men, had a lower education level, were less often alcohol users, and used less frequent psycholeptic drugs than participants without MCI.

\section{Dementia and the Risk of Cancer}

In sample 1, 1,404 (10.6\%) participants were diagnosed with dementia and 2,316 (17.5\%) individuals developed cancer, of whom 63 (4.5\%) developed cancer 


\begin{tabular}{|c|c|c|c|c|c|}
\hline & Number of persons & Number of events & & & $\mathrm{HR}(95 \% \mathrm{Cl})$ \\
\hline No dementia & 11,803 & 2,253 & & & 1.00 \\
\hline Dementia & 1,404 & 63 & $=$ & & $0.53(0.41-0.68)$ \\
\hline Alzheimer disease & 1,097 & 51 & - & & $0.52(0.39-0.69)$ \\
\hline Vascular dementia & 125 & 5 & $一-1$ & - & $0.57(0.24-1.37)$ \\
\hline After exclusion of 2 years of follow-up & 946 & 27 & - & & $0.44(0.30-0.65)$ \\
\hline After exclusion of 5 years of follow-up & 379 & 10 & $-1-$ & & $0.48(0.26-0.90)$ \\
\hline Age $<65$ years & 237 & 4 & - & & $0.22(0.08-0.58)$ \\
\hline Age $\geq 65-70$ years & 262 & 14 & -1 & - & $0.74(0.43-1.28)$ \\
\hline Age $\geq 70$ years & 905 & 45 & -- & & $0.68(0.50-0.92)$ \\
\hline Female & 996 & 36 & - & & $0.52(0.37-0.74)$ \\
\hline Male & 408 & 27 & $\rightarrow-$ & & $0.57(0.38-0.83)$ \\
\hline Non-smoker & 615 & 24 & - & & $0.52(0.34-0.80)$ \\
\hline Current smoker & 233 & 15 & -0 & & $0.58(0.34-1.00)$ \\
\hline \multirow[t]{3}{*}{ Former smoker } & 556 & 25 & - & & $0.52(0.35-0.78)$ \\
\hline & & \multirow{2}{*}{\multicolumn{4}{|c|}{$\begin{array}{ccccccc}0 & 0.5 & 1.0 & 1.5 & 2.0 & 2.5 & 3.0 \\
\mathrm{HR} & & & \end{array}$}} \\
\hline & & & & & \\
\hline
\end{tabular}

Fig. 2. Forest plot dementia and risk of cancer. HRs with $95 \%$ CIs for the risk of cancer among patients with dementia. HRs are adjusted for age, sex, body mass index, education level,

after a diagnosis of dementia. Those who developed dementia had a median follow-up time of 13.2 years (interquartile range [IQR] 10.2 years), whereas the median follow-up time for persons who were not diagnosed with dementia was 8.4 years (IQR 7.7 years). The most frequently observed cancer sites were colorectal $(15.7 \%)$, prostate $(15.5 \%)$, breast $(13.9 \%)$, and lung (11.9\%).

Dementia was associated with a decreased risk of cancer (HR 0.53; 95\% CI 0.41-0.68; Fig. 2). The risks estimates were similar for AD and vascular dementia. The risk was still reduced after excluding the first 2 and 5 years of follow-up time (HR 0.44; 95\% CI 0.30-0.65 and HR 0.48; 95\% CI 0.26-0.90, respectively). Dementia-related cancer risks did not significantly differ by age, sex, and smoking.

Reduced risks were observed when using age as time scale in the Cox model; for instance, the HR for cancer among dementia patients was 0.61 (95\% CI 0.47-0.78).

\section{MCI and the Risk of Cancer}

In sample 2, 513 (9.9\%) participants had MCI and $670(12.9 \%)$ individuals developed cancer, of whom $81(12.0 \%)$ had MCI. The median follow-up time for persons with MCI was 7.6 years (IQR 4.2 years), and 7.9 years (IQR 3.7 years) for those without MCI. A smoking status, alcohol use, and psycholeptic drug use. Characteristics are measured at time of study entry. HRs, hazard ratios. similar distribution of cancer sites was observed as in sample 1.

Individuals with MCI had a borderline with a statistically and significantly increased risk of cancer (HR 1.25; 95\% CI 0.99-1.58; Fig. 3). This increased risk was particularly pronounced for amnestic MCI (HR 1.42; 95\% CI 1.02-1.98). This risk increase was consistent when excluding the first 2 and 5 years of follow-up time (HR 1.25; 95\% CI 0.95-1.66 and HR 1.73; 95\% CI 1.19-2.51, respectively). Risk estimates for younger participants tended to be stronger compared to those of older individuals, but a formal interaction term did not reach statistical significance $(p=0.09)$. Results were comparable when using age as timescale.

The risk of cancer in patients with prevalent dementia in sample 2 was comparable to the cancer risk after incident dementia in sample 1 (HR 0.47; 95\% CI 0.21-1.06; Table 2). Risk estimates did not change materially after censoring for NMSC, stroke, or dementia (Table 2).

The abovementioned HR of cancer for MCI (1.25) was significantly different from the HR of cancer for dementia in sample $1(0.53)$ and sample $2(0.48)$, that is, persons with MCI had a significantly higher $\mathrm{HR}$ for cancer compared to persons with dementia $(p=0.001$ and $p=0.02$, respectively). 


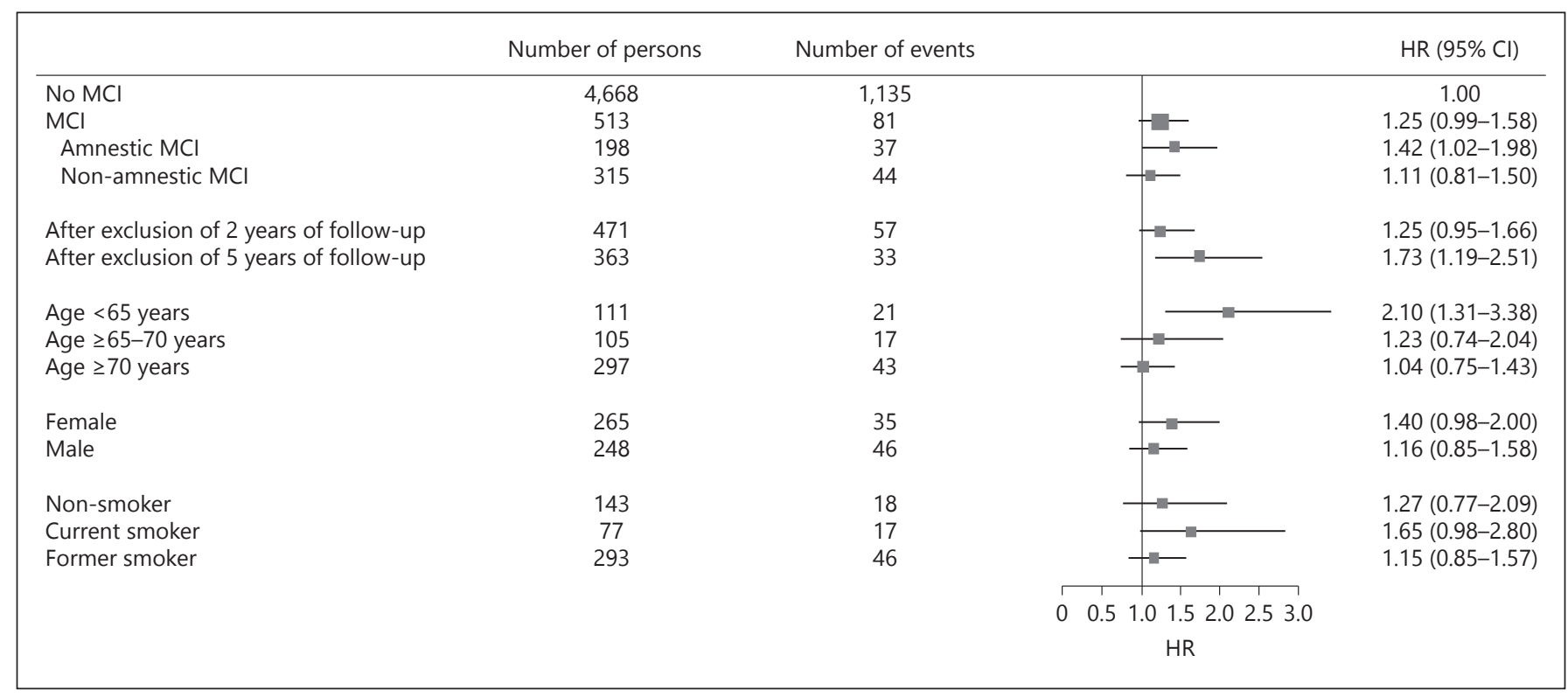

Fig. 3. Forest plot MCI and risk of cancer. HRs with 95\% CIs for the risk of cancer among patients with MCI. HRs are adjusted for age, sex, body mass index, education level, smoking status, alcohol use, and psycholeptic drug use. Characteristics are measured at the time of MCI assessment. MCI, mild cognitive impairment; HRs, hazard ratios.

Table 2. Risk of cancer in persons with MCI or prevalent dementia at the time of MCI assessment

\begin{tabular}{|c|c|c|c|c|c|}
\hline & $\begin{array}{l}\text { Number of } \\
\text { persons }\end{array}$ & \multicolumn{2}{|c|}{ Solid and hematological cancer } & \multicolumn{2}{|l|}{ Solid cancer } \\
\hline No MCI & 4,668 & 589 & 1.00 & 540 & 1.00 \\
\hline MCI & 513 & 81 & $1.25(0.99-1.58)$ & 76 & $1.29(1.02-1.65)$ \\
\hline Censored for NMSC & 483 & 74 & $1.28(1.00-1.63)$ & 69 & $1.30(1.01-1.68)$ \\
\hline Censored for stroke and dementia & 454 & 65 & $1.32(1.01-1.71)$ & 61 & $1.36(1.04-1.77)$ \\
\hline No prevalent dementia & 4,668 & 589 & 1.00 & 540 & 1.00 \\
\hline Prevalent dementia & 124 & 6 & $0.47(0.21-1.06)$ & 5 & $0.43(0.18-1.05)$ \\
\hline Censored for NMSC & 121 & 6 & $0.49(0.22-1.09)$ & 5 & $0.45(0.18-1.09)$ \\
\hline Censored for stroke & 104 & 6 & $0.60(0.27-1.35)$ & 5 & $0.56(0.23-1.35)$ \\
\hline
\end{tabular}

HRs are adjusted for age, sex, BMI, education level, smoking status, alcohol use, and psycholeptic drug use.

HR, hazard ratio; MCI, mild cognitive impairment; NMSC, non-melanoma skin cancer; BMI, body mass index.

\section{Discussion}

In this population-based cohort study, we found opposite effects of MCI and dementia with respect to subsequent risk of cancer. While we confirmed that persons with dementia had a decreased risk of cancer, those with MCI did not have a decreased risk and even tended to have an increased risk of cancer.
Strengths of our study are its prospective, populationbased design, the number of cancer diagnoses, and the standardized ascertainment of the determinants and outcome. Moreover, by focusing on MCI we were able to reduce the effect of a possible surveillance bias and the decreased life expectancy in dementia patients. In addition, we excluded the first 2 and 5 years of follow-up time in order to limit reverse causality. 
Our study has some limitations. First, the cognitive tests for assessing MCI were implemented in 2002-2005, precluding MCI assessment at baseline for RS-I and RS-II. To increase the comparability between the samples used for analyses of dementia and MCI, we investigated the risk of cancer in patients with prevalent dementia in the same sample as the MCI-analysis and we found similar results to the overall population. Second, we did not have baseline information about potential confounders such as depressive and anxiety disorders, which could have resulted in an overestimation of the observed associations between dementia and cancer, and MCI and cancer. Third, the Rotterdam Study includes mostly white, middle class persons, possibly limiting the generalizability of our findings to other ethnic and socioeconomic groups. Finally, patients with $\mathrm{AD}$ represented the largest group of people with dementia. Therefore, we were not able to reliably study the association with cancer for other dementia types.

We found that dementia is associated with a decreased risk of cancer, which is in line with findings from previous studies for various cancer types including breast, prostate, colon, and NMSC [1-8, 18]. Various biological mechanisms have been proposed, and the most frequently postulated mechanisms are to do with a genetic predisposition for either promoting or suppressing metabolic survival or apoptotic cellular pathways [10]. For instance, the tumor suppressor protein p53 induces apoptosis in the face of DNA damage, which protects against cancer, while in dementia, it could induce neuronal death [19]. Methodological explanations - like surveillance and survival bias - could also have accounted for the observed inverse relation, but these have not been sufficiently ruled out. As MCI is often considered an early manifestation of the same pathological processes as dementia and $\mathrm{AD}$, we investigated the risk of cancer among persons with MCI. We argued that if the inverse link between dementia and cancer is rooted in biology, this decreased risk would be reflected in persons with MCI as well. One previous study looked into the history of cancer among persons with and without MCI and showed that $31 \%$ of the persons with MCI were previously diagnosed with cancer [8]. However, no longitudinal analysis was performed in this group of persons and the risk of cancer after MCI or dementia diagnosis was not investigated.

In contrast to the decreased risk of cancer observed in our patients with dementia, we found that MCI was associated with an increased risk of cancer, which was borderline significant. Importantly, the difference between the risk of cancer after dementia and MCI was statistically significant. Before interpreting our results further, a word of caution is warranted. A basic premise of our study is that MCI and dementia share the same pathological underpinnings [11]. We do emphasize though that only half of MCI patients convert to dementia over a 5-year period with the other half remaining stable or even reverting back to normal, suggesting that the underlying pathology between MCI and dementia does not entirely overlap $[11,12]$. Nevertheless, 2 observations in our study support our basic premise in interpreting our findings. First, we found stronger effects for amnestic MCI than non-amnestic MCI. Indeed, amnestic MCI is more closely linked to AD pathology than non-amnestic MCI [15]. Second, censoring for dementia did not materially change the risk of cancer after MCI - if anything, the risk slightly attenuated. This suggests that those persons with MCI that went on to develop dementia (i.e., those that were censored) actually had an even higher risk of cancer than those with MCI who did not develop dementia.

The inverse link between dementia and cancer is often linked to genes involved in pathways with opposite effects in dementia and cancer. Our findings, however, point toward biological mechanisms with similar effects in both diseases. Several processes including angiogenesis, inflammation, and oxidative stress have proven to be important for tumorigenesis and there is increasing evidence that these processes also have a prominent role in the pathophysiology of $\mathrm{AD}[20,21]$. For instance, different inflammatory biomarkers are elevated in both MCI and dementia, suggesting a chronic inflammatory state [22]. Inflammatory cells can promote tumor cell growth, facilitate genomic instability, and influence tumor cell migration, and many chronic inflammatory conditions are associated with cancer [23]. Further, tumor cells can produce various cytokines and chemokines, such as tumor necrosis factor-alpha, interleukins, and interferons, to attract leukocytes and enhance inflammation. This shows that dementia and cancer could be parallel processes as a result of inflammation. Furthermore, several proteins are involved in the pathogenesis of both dementia and cancer. For instance, AD is characterized by the accumulation of plaques containing amyloid beta $(A \beta)$ peptide within the brain. It has been shown that plasma levels of $A \beta-40$ and $A \beta-42$ are increased in cancer patients [24]. Moreover, $A \beta$ precursor protein can promote cell proliferation and is increased in different types of cancer, suggesting a potential role for $A \beta$ in cancer [25]. Finally, there has been 
increasing evidence that patients with cancer have lower cognitive performance and differences in brain structure prior to cancer treatment compared to persons without cancer, indicating continuity with dementia rather than an inverse association [26, 27]. Investigation of the risk of MCI in cancer patients would therefore be very interesting, although appropriate methods should be used to deal with same potential biases as in the current study.

\section{Conclusion}

In conclusion, this is the first study to show that persons with MCI do not have a decreased risk of cancer as observed in patients with dementia, and even tended to have an increased risk. This suggests that the previously reported inverse link between dementia and cancer is based on methodological limitations. Future studies should further verify our observations and seek to eluci- date the underlying shared - instead of opposite - mechanisms between dementia and cancer. Clinically, our findings imply that for persons presenting with dementia, treating physicians should be aware of their increased risk of cancer.

\section{Acknowledgments and Funding}

This study was funded by the Dutch Cancer Society (grant number NKI-20157737). Furthermore, the Rotterdam Study is funded by Erasmus Medical Center and Erasmus University, Rotterdam, Netherlands Organization for the Health Research and Development (ZonMw), the Research Institute for Diseases in the Elderly, the Ministry of Education, Culture and Science, the Ministry for Health, Welfare and Sports, the European Commission (DG XII), and the Municipality of Rotterdam. The funders had no role in study design, data collection and analysis, decision to publish, or preparation of the manuscript.

We gratefully thank all Rotterdam Study participants and staff for their time and commitment.

\section{References}

1 Driver JA, Beiser A, Au R, Kreger BE, Splansky GL, Kurth T, Kiel DP, Lu KP, Seshadri S, Wolf PA: Inverse association between cancer and Alzheimer's disease: results from the framingham heart study. BMJ 2012; 344:e1442.

2 Musicco M, Adorni F, Di Santo S, Prinelli F, Pettenati C, Caltagirone C, Palmer K, Russo $\mathrm{A}$ : Inverse occurrence of cancer and Alzheimer disease: a population-based incidence study. Neurology 2013;81:322-328.

3 Ou SM, Lee YJ, Hu YW, Liu CJ, Chen TJ, Fuh JL, Wang SJ: Does Alzheimer's disease protect against cancers? A nationwide populationbased study. Neuroepidemiology 2013;40:4249.

4 Realmuto S, Cinturino A, Arnao V, Mazzola MA, Cupidi C, Aridon P, Ragonese P, Savettieri G, D'Amelio M: Tumor diagnosis preceding Alzheimer's disease onset: is there a link between cancer and Alzheimer's disease? J Alzheimers Dis 2012;31:177182.

5 Roe CM, Fitzpatrick AL, Xiong C, Sieh W, Kuller L, Miller JP, Williams MM, Kopan R, Behrens MI, Morris JC: Cancer linked to Alzheimer disease but not vascular dementia. Neurology 2010;74:106-112.

6 Romero JP, Benito-Leon J, Louis ED, Bermejo-Pareja F: Alzheimer's disease is associated with decreased risk of cancer-specific mortality: a prospective study (nedices). J Alzheimers Dis 2014;40:465-473.

7 Shi HB, Tang B, Liu YW, Wang XF, Chen GJ: Alzheimer disease and cancer risk: a meta- analysis. J Cancer Res Clin Oncol 2015;141: 485-494.

8 Nudelman KN, Risacher SL, West JD, McDonald BC, Gao S, Saykin AJ; Alzheimer's Disease Neuroimaging Initiative: Association of cancer history with Alzheimer's disease onset and structural brain changes. Front Physiol 2014;5:423.

9 Behrens MI, Lendon C, Roe CM: A common biological mechanism in cancer and Alzheimer's disease? Curr Alzheimer Res 2009;6:196204.

10 Driver JA: Inverse association between cancer and neurodegenerative disease: review of the epidemiologic and biological evidence. Biogerontology 2014;15:547-557.

11 Petersen RC, Doody R, Kurz A, Mohs RC, Morris JC, Rabins PV, Ritchie K, Rossor M, Thal L, Winblad B: Current concepts in mild cognitive impairment. Arch Neurol 2001;58: 1985-1992.

12 Gauthier S, Reisberg B, Zaudig M, Petersen RC, Ritchie K, Broich K, Belleville S, Brodaty $H$, Bennett $D$, Chertkow $H$, Cummings JL, de Leon M, Feldman H, Ganguli M, Hampel H, Scheltens P, Tierney MC, Whitehouse P, Winblad B; International Psychogeriatric Association Expert Conference on mild cognitive impairment: Mild cognitive impairment. Lancet 2006;367: 1262-1270.

13 Ikram MA, Brusselle GG, Murad SD, van Duijn $\mathrm{CM}$, Franco $\mathrm{OH}$, Goedegebure A, Klaver CC, Nijsten TE, Peeters RP, Stricker $\mathrm{BH}$, Tiemeier H, Uitterlinden AG, Vernooij
MW, Hofman A: The Rotterdam Study: 2018 update on objectives, design and main results. Eur J Epidemiol 2017;32:807-850.

14 de Bruijn RF, Bos MJ, Portegies ML, Hofman A, Franco OH, Koudstaal PJ, Ikram MA: The potential for prevention of dementia across two decades: the prospective, population-based Rotterdam Study. BMC Med 2015;13:132.

15 de Bruijn RF, Akoudad S, Cremers LG, Hofman A, Niessen WJ, van der Lugt A, Koudstaal PJ, Vernooij MW, Ikram MA: Determinants, MRI correlates, and prognosis of mild cognitive impairment: the Rotterdam Study. J Alzheimers Dis 2014;42(suppl 3):S239-S249.

16 Muka T, Kraja B, Ruiter R, Lahousse L, de Keyser CE, Hofman A, Franco OH, Brusselle G, Stricker BH, Kiefte-de Jong JC: Dietary mineral intake and lung cancer risk: he Rotterdam Study. Eur J Nutr 2017;56:1637-1646.

17 Ullen H, Augustsson K, Gustavsson C, Steineck G: Supplementary iron intake and risk of cancer: reversed causality? Cancer Lett 1997;114:215-216.

18 Schmidt SA, Ording AG, Horvath-Puho E, Sorensen HT, Henderson VW: Non-melanoma skin cancer and risk of Alzheimer's disease and all-cause dementia. PLoS One 2017; 12:e0171527.

19 Cenini G, Sultana R, Memo M, Butterfield DA: Elevated levels of pro-apoptotic p53 and its oxidative modification by the lipid peroxidation product, HNE, in brain from subjects with amnestic mild cognitive impairment and Alzheimer's disease. J Cell Mol Med 2008;12: 987-994. 
20 Vagnucci AH Jr, Li WW: Alzheimer's disease and angiogenesis. Lancet 2003;361:605608.

21 Licastro F, Candore G, Lio D, Porcellini E, Colonna-Romano G, Franceschi C, Caruso C: Innate immunity and inflammation in ageing: a key for understanding age-related diseases. Immun Ageing 2005;2:8.

22 Trollor JN, Smith E, Baune BT, Kochan NA, Campbell L, Samaras K, Crawford J, Brodaty $\mathrm{H}$, Sachdev P: Systemic inflammation is associated with MCI and its subtypes: the sydney memory and aging study. Dement Geriatr Cogn Disord 2010;30:569-578.
23 Coussens LM, Werb Z: Inflammation and cancer. Nature 2002;420:860-867.

24 Jin WS, Bu XL, Liu YH, Shen LL, Zhuang ZQ, Jiao SS, Zhu C, Wang QH, Zhou HD, Zhang T, Wang YJ: Plasma amyloid-beta levels in patients with different types of cancer. Neurotox Res 2017;31:283-288.

25 Lim S, Yoo BK, Kim HS, Gilmore HL, Lee Y, Lee HP, Kim SJ, Letterio J, Lee HG: Amyloid- $\beta$ precursor protein promotes cell proliferation and motility of advanced breast cancer. BMC Cancer 2014;14:928.
26 Kesler SR, Adams M, Packer M, Rao V, Henneghan AM, Blayney DW, Palesh O: Disrupted brain network functional dynamics and hyper-correlation of structural and functional connectome topology in patients with breast cancer prior to treatment. Brain Behav 2017;7:e00643.

27 Patel SK, Wong AL, Wong FL, Breen EC, Hurria A, Smith M, Kinjo C, Paz IB, Kruper L, Somlo G, Mortimer JE, Palomares MR, Irwin MR, Bhatia S: Inflammatory biomarkers, comorbidity, and neurocognition in women with newly diagnosed breast cancer. J Natl Cancer Inst 2015; 107:pii:djv131. 